\title{
REM restriction persistently alters strategy used to solve a spatial task
}

\author{
Theresa E. Bjorness, ${ }^{1}$ Brett T. Riley, ${ }^{2}$ Michael K. Tysor, ${ }^{2}$ and Gina R. Poe ${ }^{2,3,4}$ \\ ${ }^{1}$ Neuroscience Program, ${ }^{2}$ Department of Anesthesiology, and ${ }^{3}$ Department of Molecular and Integrative Physiology, University of \\ Michigan, Ann Arbor, Michigan 48014, USA
}

\begin{abstract}
We tested the hypothesis that rapid eye movement (REM) sleep is important for complex associative learning by restricting rats from entering REM sleep for $4 \mathrm{~h}$ either immediately after training on an eight-box spatial task (0-4 REMr) or $4 \mathrm{~h}$ following training (4-8 REMr). Both groups of REM-restricted rats eventually reached the same overall performance level as did nonrestricted controls, but 0-4 REMr animals were delayed in their improvement in the first few days and lagged behind controls in the middle portion of the training period. More importantly, performance gains of 0-4 REMr rats depended more on simple local cues throughout the 15-d study since, unlike control and 4-8 REMr animals, their error rate increased after daily disruption of the relationship between local (intramaze) cues and the food reward. Thus, although overall performance was only subtly and transiently impaired, due to the ability to use alternate, nonspatial behavioral strategies, complex associative (spatial) learning was persistently impaired by restricting REM for a short critical period each day.
\end{abstract}

\section{Memory systems and REM sleep}

While evidence suggests that people remember fewer details of previous experience after a night of little or no sleep (Dinges et al. 1997; Van Dongen et al. 2003), the nature of the dependence of learning and memory on sleep is largely unknown. Much of the difficulty in making a strong argument for the role of sleep in learning (Vertes and Eastman 2000; Siegel 2001) stems from the often temporary and subtle variations in performance exhibited after sleep deprivation. For example, people sleeping relatively few hours per night can still adequately perform most workrelated functions most of the time, especially with the use of a stimulant (Bonnet and Arand 1994; Westensten et al. 2002; Wyatt et al. 2004). Sleep deprivation increases the areas and number of brain regions activated in completing a complex or split-attention task (Drummond et al. 2000; Drummond et al. 2001). As in the case when an area of the brain normally involved in a task is compromised, other brain regions may be invoked to compensate. For example, when age or local anesthetic reduces hippocampal function, performance on a spatial task can remain adequate by the use of working memory rehearsal or nonhippocampus-dependent cue strategies (Barnes et al. 1980; Rapp et al. 1987; Poe et al. 2000a). Under sleep deprivation, the increased brain response may allow people to function normally until a crisis or critical multifactorial decision point arrives. At this point the reserve processing areas, already tapped for normal processing, cannot support the additional load and judgment can be disastrously impaired. Lack of sleep was implicated in four different nuclear power plant accidents and near accidents as well as in decisions surrounding disastrous and near disastrous space shuttle launches (Mitler et al. 1988). Thus, although overall performance may be only mildly impaired or not impaired at all under standard conditions, high-level processing mechanisms remain dysfunctional with total sleep or selective rapid eye movement (REM) deprivation.

A preponderance of evidence suggests that sleep is important for certain types of complex learning requiring the fast as-

\section{${ }^{4}$ Corresponding author.}

E-mail ginapoe@umich.edu; fax (734) 764-9332.

Article published online ahead of print. Article and publication date are at http://www.learnmem.org/cgi/doi/10.1101/lm.84805. sociation between different processing regions. Spatial learning, which associates the sight, sound, and the many contextual cues that identify the place of a reward, is dependent on the hippocampus (O'Keefe and Nadel 1978; Vargha-Khadem et al. 1997; Eichenbaum 2000) and seems to require sleep, specifically REM sleep. Both total sleep deprivation and selective REM sleep deprivation result in performance impairments on spatial tasks in rodents (Youngblood et al. 1997; Smith et al. 1998; Guan et al. 2004) and on various cognitive, perceptual, and motor tasks in humans (Wimmer et al. 1992; Karni et al. 1994; Stickgold et al. 2001).

In accordance with results from a number of experiments (Seligman 1970; Greenberg and Pearlman 1974; McGrath and Cohen 1978; Pearlman 1979; Winson 1985), we hypothesize that REM sleep is important for complex associative learning, such as spatial learning. For spatial learning, distal allocentric (global environmental) cues are used more than are proximal egocentric cues (Shapiro et al. 1997). Learning to use allocentric cues requires the hippocampus. When the hippocampus is damaged either permanently or temporarily, tasks are solved by using nonspatial adaptive strategies, such as procedural strategies (DiMattia and Kesner 1988). For example, rats with hippocampal damage can perform the Morris water maze task by swimming around the tank at a fixed distance from the perimeter to find the hidden platform (Morris et al. 1982). This thigmotaxic strategy is less efficient than a spatial strategy in which animals swim directly to the hidden platform location no matter where they are originally placed within the tank (DiMattia and Kesner 1988). If our hypothesis that REM sleep is important for complex associative learning is correct, then REM restriction while learning a spatial task should impair spatial learning while enhancing the use of less-efficient alternate strategies. We tested our hypothesis with a task that simultaneously measures the use of spatial solutions (using extramaze, allocentric cues) against the use of more simple (intramaze, egocentric) cues to see if REM sleep restriction differentially impairs complex associative learning.

\section{REM sleep timing and learning}

REM sleep may need to occur at specific times following training in order to effectively facilitate learning. REM sleep restriction 
immediately following training in rats disrupts spatial learning on the eight-arm radial maze (Smith et al. 1998) and on the Morris water maze (Smith and Rose 1997). REM sleep expression is not uniform in timing and/or duration across the sleep cycle in both rats (Borbely and Neuhaus 1979; Borbely 1980; Wurts and Edgar 2000) and humans (Feinberg and Floyd 1979), allowing for the possibility that REM during one portion of the sleep cycle may be more or less important for the retention or consolidation of newly learned information. The time period when REM sleep restriction significantly impairs learning coincides with spontaneous increases in REM sleep subsequent to the training sessions (rats, Smith and Butler 1982; Smith 1985; humans, De Koninck et al. 1989) and has been called the "REM window." When application of a protein synthesis inhibitor or an acetylcholine antagonist, both of which impair memory (Eckerman et al. 1980; Okaichi et al. 1989; Meiri and Rosenblum 1998; Naghdi et al. 2003), coincides with the beginning of the REM window, shuttle box learning is impaired in rats (Smith et al. 1991). The REM window for intensive training (e.g., many trials over a short period of time) tends to occur in the first few hours of sleep after learning (Smith 1985; Smith and Rose 1996), while the REM window for fewer trials occurs relatively later, according to animal studies (Smith 1985). This REM window phenomenon may explain why researchers have mixed results when using REM deprivation at various times post-training (for review, see Smith 1985). If the REM window concept is pertinent to complex associative learning, then total overall REM sleep deprivation should not be necessary to impair learning. Restricting animals from entering REM in key hours, even if the total REM sleep time is unchanged, should be enough to impede the function of REM for learning. We tested this idea by not allowing animals to enter REM only for the first $4 \mathrm{~h}$ after training each day, or by allowing them to sleep normally for $4 \mathrm{~h}$ following training, then restricting them from REM in the second 4 -h period.

In a preliminary study we found that, unlike control animals, the performance of rats deprived of REM sleep for $4 \mathrm{~h}$ after training did not reach asymptote (no performance improvement across three consecutive days) within a 6-d experimental period. In the present study, we continued daily training until REMrestricted and control groups reached performance asymptote to see whether REM-restricted animals could reach the same overall performance level as controls, and if so, how long it would take each group to reach that level. Finally, to see if REM-restricted animals ever learned the complex associative spatial task, we assessed the various performance strategies of the rats with procedures that tested simple cue use versus complex spatial learning over the entire 15-d learning period.

\section{Results}

Three groups of rats were trained on a spatial learning task, the eight-box task (for task description, see Materials and Methods) (Fig 6 below; Poe et al. 2002), for $30 \mathrm{~min} / \mathrm{d}$ for $15 \mathrm{~d}$. Each day, after training, rats in one group (control) were returned to their home cage, while two groups were deprived of REM sleep for $4 \mathrm{~h}$ either immediately after training (0-4 REMr) or $4 \mathrm{~h}$ after training (4-8 REMr). Relatively selective REM sleep deprivation was accomplished by the standard multiple platform over water method (see Materials and Methods).

Comparison of performance in all three groups showed 0-4 REMr animals to be significantly different from controls on most tests, whereas 4-8 REMr animals performed at intermediate levels, not significantly different from either of the two other groups. Therefore, for clarity, results for the $4-8 \mathrm{REMr}$ group are presented in the "Time of REM Deprivation" section toward the end of the Results section, while the other sections strictly compare the 0-4 REMr and control groups.

\section{Learning curve (errors per lap)}

All rats started the experiment making approximately four errors per lap on the novel maze. In the first 5-d segment, there was no difference in performance between groups $(P=0.526)$ (Fig. $1 \mathrm{~A}$, segment 1$)$. Presumably the availability of food within the corncob bedding of all the rats in the first $4 \mathrm{~d}$ was responsible for delayed weight loss and the few laps completed each day (see Fig. 4 below). Overall performance also did not significantly differ between groups during the final 5-d segment of the experiment $(P=0.132)$ (Fig. 1A, segment 3$)$. Thus, across the entire 15-d period there was no overall significant difference in errors per lap between the groups $(P=0.162)$. However, in the mid portion of the experiment (days 6-10), controls committed significantly fewer errors per lap $(P=0.052)$ than did animals restricted from entering REM in the first $4 \mathrm{~h}$ after training (Fig. 1A, segment 2). The control group reached criterion (less than one error per lap) and maintained performance by day 10 onward, after completing an average of 108.8 laps. The $0-4$ REMr group reached criterion and asymptote $3 \mathrm{~d}$ later, after completing an average of 139.5 laps (lap effect between groups, $P=0.421$ ).

\section{Improvement}

Similar to the overall performance levels, controls showed a higher rate of improvement in the mid portion of the experiment did than $0-4$ REMr rats $(P=0.024)$ (Fig. 1B, segment 2$)$. Improved performance from baseline remained higher for controls than
A

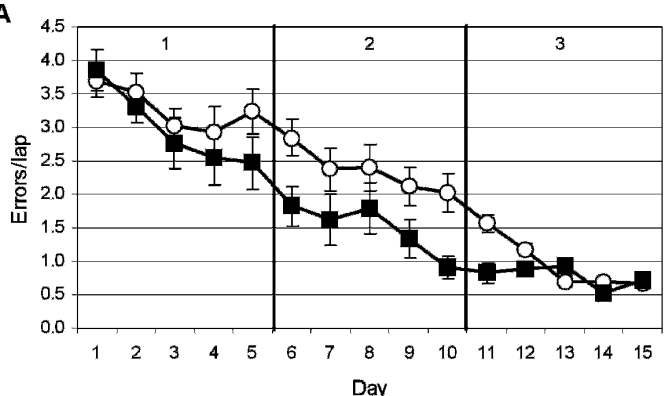

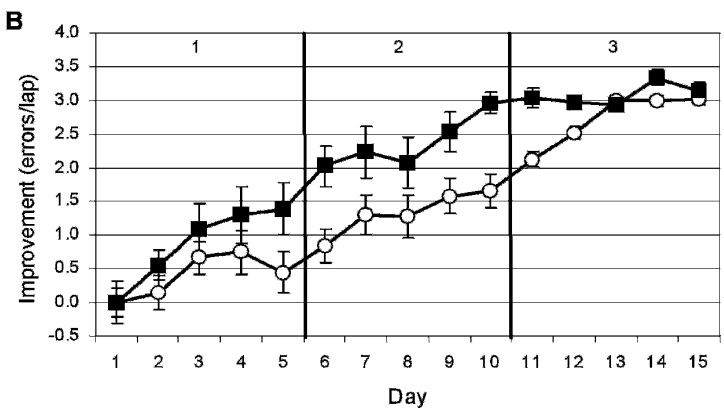

Figure 1. Learning $(A)$ and improvement $(B)$ curves for the 15-d experiment. $(A)$ For the learning curve the mean number of errors committed per lap are plotted against day of training for controls (solid squares) and 0-4 REMr (open circles). Error bars indicate SEM. Controls committed fewer errors during the middle segment of the study (second 5-d segment; 2 ) than did the 0-4 REMr group. No significant difference in performance was seen between groups in either the first 5-d segment (1) or the third 5-d segment (3) of the study. (B) Improvement is calculated by subtracting each day's mean number of errors per lap from the group average number of errors per lap on day 1 . The $0-4$ REMr group (open circles) showed less improvement than did controls (solid squares) during segments 2 and 3 of the study. 
for 0-4 REMr animals in the last portion of the experiment as well $(P=0.008)$ (Fig. 1B, segment 3$)$. Regression analysis showed the slope of improvement to be greatest for controls during the first segment ( $r=0.352$; day effect, $P=0.011)$, then decreasing over the second $(r=0.216$; day effect, $P=0.045)$ and third segments $(r=0.059$; no significant day effect, $P=0.327)$. Over the entire experiment, the slope of improvement for controls was 0.220 (day effect, $P<0.001$ ). The $0-4$ REMr animals had a similar overall slope of improvement $(r=0.228$; day effect, $P<0.001)$. However, unlike controls, 0-4 REMr animals showed the greatest rate of improvement during the second and third segments of training $(r=0.191$; day effect, $P=0.016$ and $r=0.226$; day effect, $P=0.039$, respectively). Improvement rate was lower during the first segment $(r=0.149$; no significant day effect, $P=0.16)$. Controls tended to have a steeper slope and therefore improved more than 0-4 REMr animals during the first segment $(P=0.093)$, while 0-4 REMr animals tended to have a steeper slope and improved more than did controls during the third segment $(P=0.087)$. Thus, the pattern of improvement varied between groups, with controls showing larger day-to-day improvement in the early part of the learning curve and $0-4$ REMr showing little improvement early on, and most improvement later.

\section{Error types}

Three types of errors were scored. Across days, errors of commission (EC; when an animal investigated an unbaited box) were greater than the number of errors of hesitation (EH; when an animal hesitated at an unbaited box) or of omission (EO; when an animal ignored a baited box) for both groups (0-4 REMr, $P<0.001$; control, $P<0.001$ ) (Fig. 2). Errors of commission primarily accounted for the difference in errors per lap between $0-4$ REMr and control groups. The number of commission errors had a significant day-by-group interaction $(P=0.031)$. Though the number of laps completed increased in both groups after the first $5 \mathrm{~d}$ (see Fig. 4 below), the number of errors of commission committed by controls did not change whereas they rose in 0-4 REMr animals in the second segment and were significantly different from control levels on day $10(P=0.009)$ and day $11(P=0.007)$. In the third segment, the number of errors of commission committed by $0-4$ REMr animals decreased to control levels.

In analyses of both errors of hesitation and omission, there was a significant effect of day (hesitation, $P=0.009$; omission, $P<0.001$ ) (Fig. 2B,C) but not of group (hesitation, $P=0.745$; omission, $P=0.966$ ). That is, both groups committed more errors of omission in the beginning before they learned that the nature of the task was to check boxes for food rewards, and increased their errors of hesitation in the middle portion as they learned the task procedure of checking boxes but remained uncertain of the food box placements.

\section{Improvement within and between days}

Although the large variance in performance day to day washed out group differences with ANOVA comparisons, on 14 out of 15 d, 0-4 REMr animals committed a higher number of errors through the first five laps compared with controls (Sign test, $P=0.005$ ) (Fig. 3A). There was no significant difference between groups for the remaining lap sets (laps 6-10 and laps 11-15), although the low number of laps completed early in training reduced the number of days included in the analysis ( $7 \mathrm{~d}$ for laps 6-10; $6 \mathrm{~d}$ for laps 11-15). All rats improved performance within a practice session (Fig. 3B), but control animals started most days with fewer errors and, so, retained more information about the location of food boxes between days.

\section{Weight}

Across the 15-d experiment, body weight decreased significantly in both $0-4$ REMr and control groups $(P<0.001)$ (Fig. 4). There
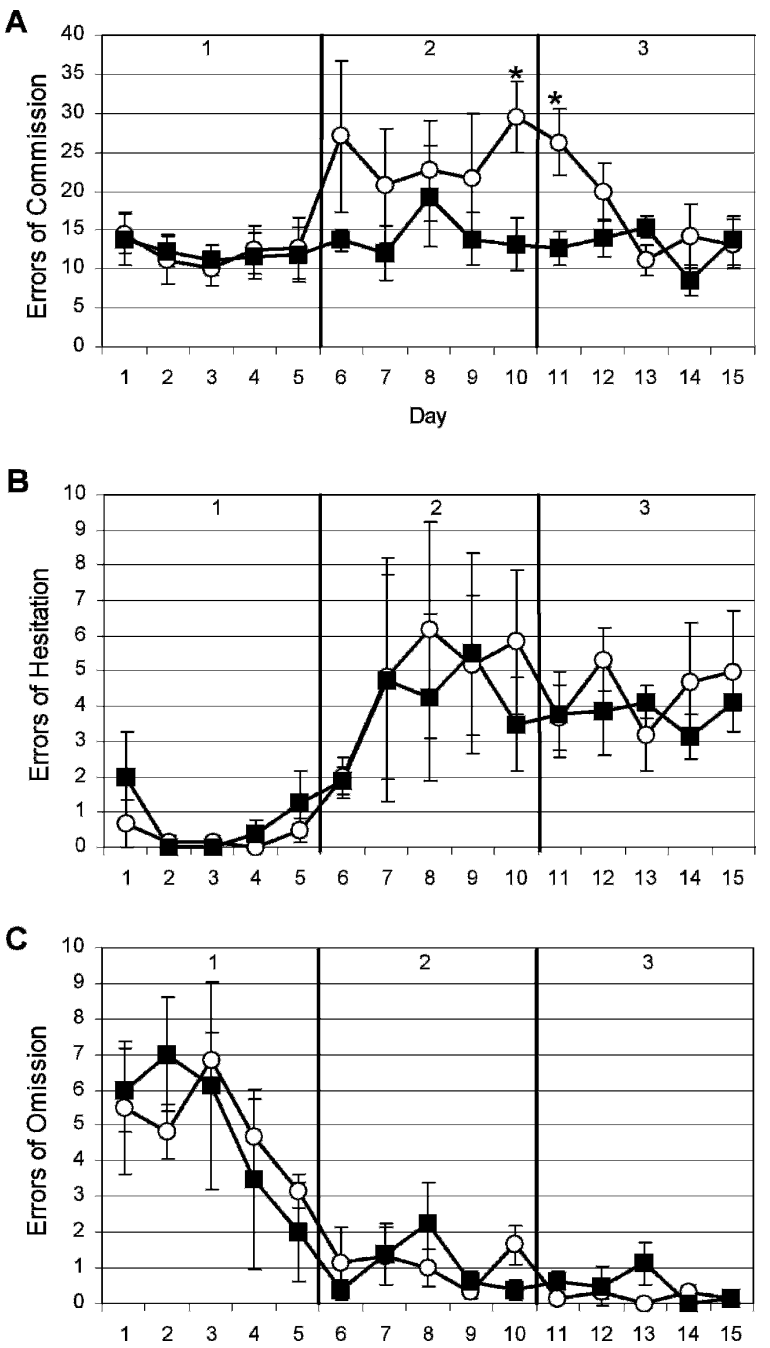

Figure 2. (A) Errors of commission across days. Errors of commission accounted for the differences in learning and improvement curves (Fig. 1 A,B) between 0-4 REMr (open circles) and controls (solid squares). There was a significant difference in errors of commission between groups on day 10 and day 11 , as indicated by an asterisk $(P<0.01)$. Errors of hesitation $(B)$ and errors of omission $(C)$ showed no difference between groups across days.

was no day-by-group interaction. In the first and second 5-d segments, there was also no difference in weight between groups (segment $1, P=0.248$; segment $2, P=0.312$ ), but in the third segment, controls weighed slightly more than did 0-4 REMr animals $(P=0.046)$.

\section{Number of laps completed}

There was a significant increase in the number of laps completed across the 15 -d experiment in both groups $(P<0.001)$ (Fig. 4$)$. However, there was no difference in the number of laps completed each day between groups $(P=0.983)$. Additionally, there was no group difference in laps completed daily in any of the 5-d segments (segment $1, P=0.429$; segment $2, P=0.683$; segment 3 , $P=0.544$ ). Both groups averaged approximately five laps per day for the first $5 \mathrm{~d}$. Laps completed increased dramatically over the next $5 \mathrm{~d}$. By day 15, rats averaged $\sim 25$ laps for the session.

\section{Time of REM deprivation}

As noted earlier, animals restricted from entering REM sleep $4 \mathrm{~h}$ after training (4-8 REMr) performed at an intermediate level be- 

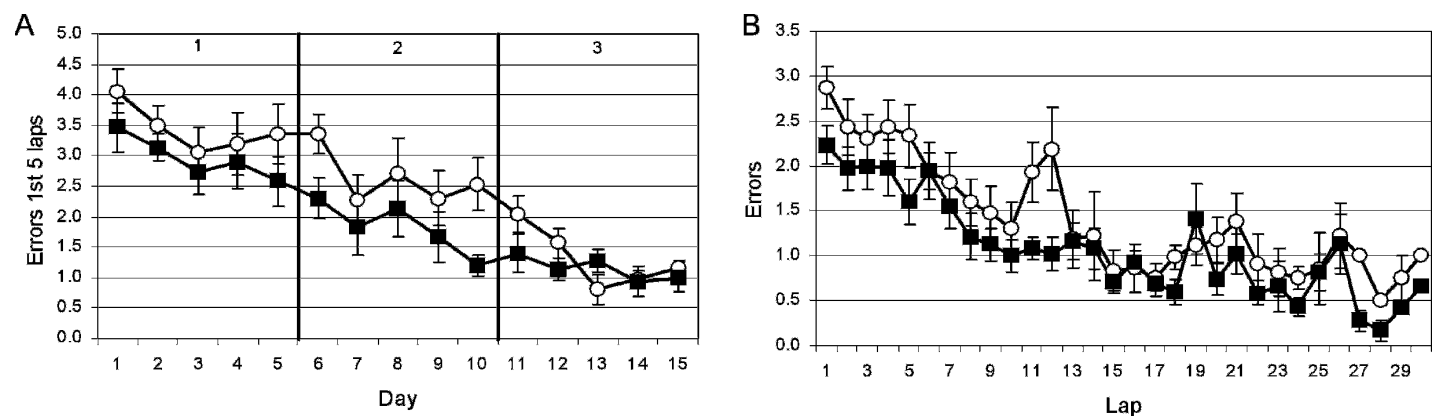

Figure 3. (A) Errors per lap on first five laps each day. Rats in the 0-4 REMr group (open circles) committed a higher average number of errors per lap on the initial five laps on 13 of $15 \mathrm{~d}$ compared with controls (solid squares). (B) Errors across laps. Both groups showed a decrease in average errors committed from the beginning of the session (lap 1) to the end of the session (lap 30) across days. Errors each lap were averaged across days, with the cutoff set at lap 30 as fewer than two rats per group were completing $>30$ laps. The $0-4$ REMr group increased errors on laps 11 and 12 , indicating an effect of disruption due to maze rotation (see Materials and Methods).

tween 0-4 REMr and control groups for most measures. However, on 13 of $15 \mathrm{~d}$, rats in the $0-4$ REMr group committed more errors in the first five laps of the training period than did the 4-8 REMr group ( $P=0.003$ ) (Fig. 5A). The 4-8 REMr group weighed less on average across the $15-\mathrm{d}$ training period $(0-4 \mathrm{REMr}, 280.4 \pm 5.5 \mathrm{~g}$; 4-8 REMr, $254.2 \pm 6.1 \mathrm{~g} ; P=0.015$ ). There was no difference between the two REM-restricted groups in errors per lap across 15 $\mathrm{d}(P=0.867)$ or within the three 5 -d segments (segment 1 , $P=0.761$; segment $2, P=0.953$; segment $3, P=0.803$ ). There was also no difference between REM-restricted groups in improvement $(P=0.841)$, types of errors committed (EC, $P=0.492$; $\mathrm{EH}$, $P=0.824$; EO, $P=0.655)$, or number of laps completed each day $(P=0.488)$. There was no significant difference in the number of laps needed to reach criterion (0-4 REMr, 139.6; 4-8 REMr, 107.2; $P=0.47)$. One rat in the $4-8$ REMr group, however, never performed to criterion.

\section{Strategy used to find food}

To determine whether animals relied on a spatial or nonspatial strategy to solve the task, two types of probe tests were employed. To reveal local, simple cue use, the maze was rotated $180^{\circ}$ following lap 10, and the same box positions relative to the room (different physical boxes) were baited. To reveal thigmotaxic strategies, rats were moved after every five laps to a resting pot for 2 min and then replaced at semirandom positions on the maze. The performance on lap 10 (before maze rotation) was compared to performance on lap 11 (following maze rotation). A decrease

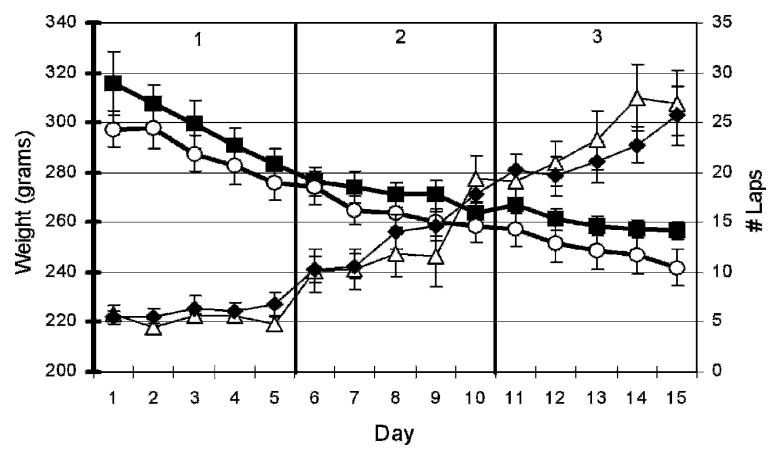

Figure 4. Rat weight and number of laps completed across days. Weight (left axis) decreased for both 0-4 REMr (open circles, thick line) and controls (solid squares, thick line) across the experiment. Controls were significantly heavier during the third 5-d segment. Number of laps completed (right axis) increased for both 0-4 REMr (open triangles, thin line) and controls (solid diamonds, thin line) across the experiment, with no group difference. or no change in errors between these two laps suggests the animals relied on a spatial strategy since the relative food box positions to extramaze cues remained the same. On the other hand, an increase in errors on lap 11 suggests animals relied on nonspatial strategies since the food box positions relative to intramaze cues deposited by rats within a session (such as urine, food crumbs, etc.) changed with maze rotation. While both 4-8 REMr and control groups showed no difference in average errors between laps 10 and 11 (Sign test, 4-8 REMr: lap 10, $1.08 \pm 0.4$; lap $11,1.04 \pm 0.4 ; P=0.31$; control: lap $10,0.52 \pm 0.12$; lap 11 , $0.96 \pm 0.14 ; P=0.09$ ) (Fig. 5C,D), the average errors committed by the 0-4 REMr group on lap 11 increased compared with lap 10 (lap 10, $0.75 \pm 0.19$; lap 11, $1.33 \pm 0.2 ; P=0.02$ ) (Figs. 5B, 3B). There was no difference in errors committed between laps 5 and 6 (when the animal had a 2-min break and was replaced on the track) for the 0-4 REMr group $(P=0.226)$, indicating that removal from and replacement on the track in a different location is not responsible for the performance disruption on lap 11. The 4-8 REMr group also did not show a difference in errors committed on lap 5 versus lap $6(P=0.205)$; however, the control group showed a slight trend of increased errors on lap $6(P=0.081)$. The lack of disruption with random placement on the maze combined with the errors after uncoupling local and global cues (maze rotation after lap 10) suggests that 0-4 REMr animals were relying on simple, intramaze cues rather than using an allocentric spatial strategy to find food. Controls and 4-8 REMr animals were not disrupted by maze rotation, indicating that they relied on a spatial strategy.

\section{Discussion}

REM restriction for $4 \mathrm{~h} / \mathrm{d}$ immediately following a learning period caused a subtle and temporary deficit in overall performance of the eight-box spatial task. However, the 0-4 REMr group performed worse than did controls and 4-8 REMr animals on the first five laps for 13 of $15 \mathrm{~d}$ of training, indicating that early REM restriction interfered with long-term maintenance of reference memory. On day 1 , before the first REMr procedure was performed, the 0-4 REMr group showed a slightly higher average number of errors per lap on the first five laps compared with the control and 4-8 REMr groups. Although the difference did not reach significance, if the group happened to be initially impaired compared with the other groups, it could explain the relative impairment of the 0-4 REMr group. However, in a prior study the control group committed a higher number of errors per lap across the first five laps on the first day, yet outperformed REMrestricted animals in the long term (Poe et al. 2000b). Thus, the first day effect in the present study should not account for the continued impairments of the $0-4$ REMr group. 

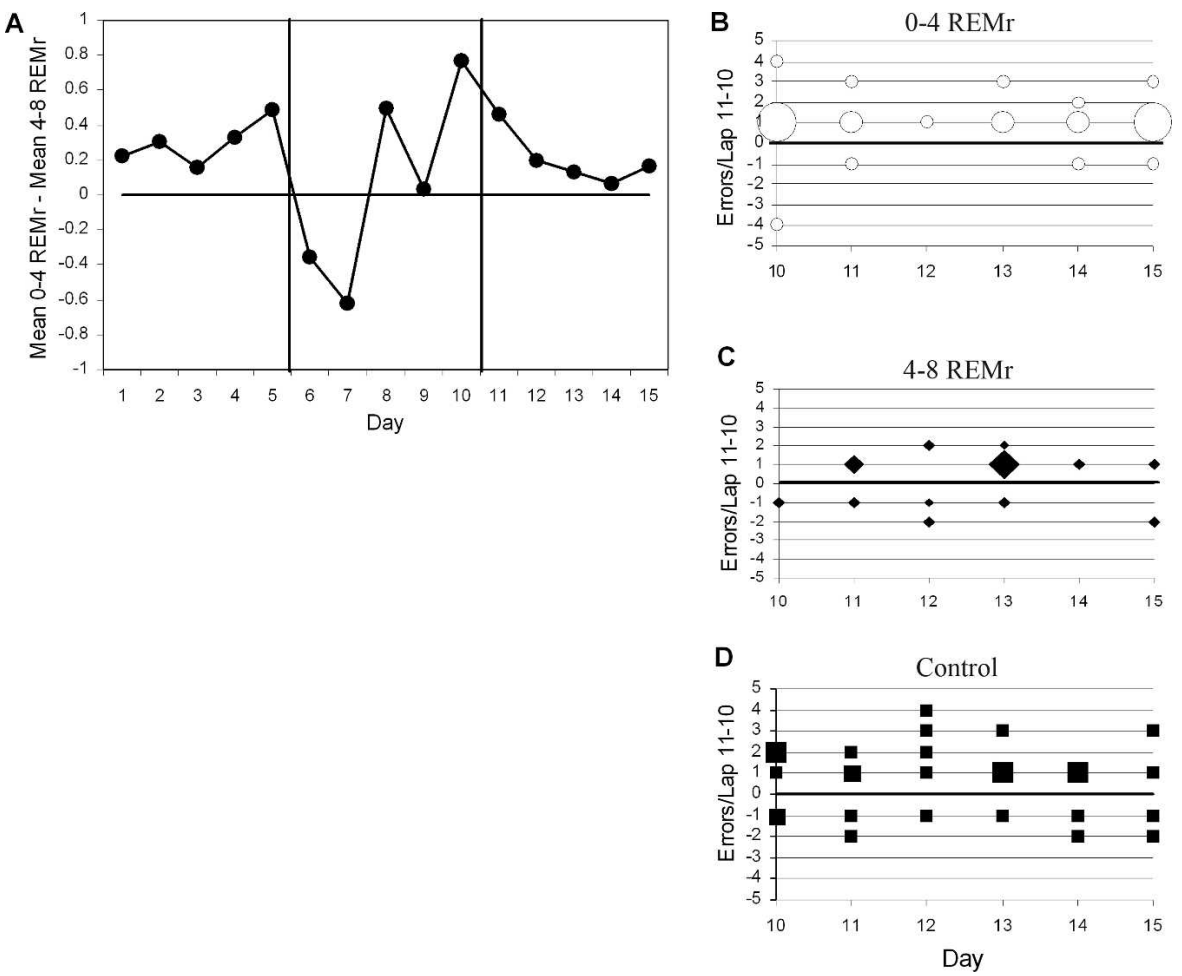

Figure 5. (A) Difference in average errors per lap on the first five laps between 0-4 and 4-8 REMr groups. The average errors per first five laps of 4-8 REMr animals were subtracted from the average errors per first five laps of $0-4 \mathrm{REMr}$ animals each day. The zero line indicates equivalent performance. On 13 of $15 \mathrm{~d}, 0-4 \mathrm{REMr}$ rats had a higher average number of errors on the first five laps. $(B-D)$ Performance prior to and following maze rotation. The number of errors on lap 10 was subtracted from the number of errors on lap 11 to show an increase (positive numbers) or decline (negative numbers) in errors after the maze was rotated $180^{\circ}$. Small symbols depict one animal, medium sized symbols depict two animals, and large symbols depict three animals. For clarity, points falling on the zero line were omitted. The $0-4 \mathrm{REMr}$ group $(B)$ had a majority of points greater than equivalency, indicating an increase in errors after maze rotation. The 4-8 REMr group (C) and control group $(D)$ have a more equal distribution of points, indicating no overall effect of maze rotation.

Animals with early REM sleep restriction also continued to make more errors after maze rotation each day (lap 11) through the end of the study, even after their overall daily performance reached asymptote. Thus, $4 \mathrm{~h}$ of REM restriction immediately following learning seems to have increased the use of alternate, nonspatial, cue-based learning strategies. Early REM restriction did not affect the use of working memory within a day since, after the "reminder" of the first few laps of the day and after the first few laps following maze rotation each day, there was no difference in errors per lap between groups (Fig. 3B). REM sleep restriction also did not affect the rate of learning the procedures of the task, since the pattern of errors of hesitation and omission were the same between REM-restricted and control groups. Errors of omission generally occurred early in training when the rats were still learning that food was present in three boxes. Errors of hesitation increased as the rats learned that food was available in some boxes. It is perhaps a misnomer to call hesitations errors, but we felt it important to include these pausing behaviors as they probably reflected uncertainty as to exactly which boxes contained food, but showed they learned the procedure of checking boxes for food.

A selective effect on reference versus simple cued memory fits with results of REM deprivation in several other studies (Youngblood et al. 1997; Smith et al. 1998; Ruskin et al. 2004). The ability of rats to use alternative strategies to solve spatial tasks has heretofore made it difficult to make strong conclusions about the role of REM sleep in learning. The use of alternate pared with that of controls, similar to the alteration observed in aged rats who are also relatively impaired on spatial tasks (Barnes et al. 1997).

\section{REM sleep timing relative to training}

Rats in the 4-8 REMr group differed from those in the 0-4 REMr group in that they seemed to rely on a spatial strategy to a similar degree as controls. They were not disrupted by maze rotation, and they performed better than did early REM-restricted rats on the first few laps each day. This difference in strategies between REM-restricted groups may indicate that REM sleep immediately following training is more important for learning the spatial solution to this task. In order to draw strong conclusions regarding the importance of REM sleep timing, groups of rats restricted from entering REM sleep at other windows, e.g., 12-16 $\mathrm{h}$ after training, are needed. Also, the spontaneous increase in REM sleep that is seen after training on other tasks (Lucero 1970; Fishbein et al. 1974; Smith and Rose 1997) could be measured to determine the most critical time of REM sleep for this task, which may change as the number of laps completed each day rises.

\section{Motivation}

There was no difference in the number of total laps completed per group, ruling out the possibility that control rats had more practice within a day. Weight loss also did not correlate with performance, suggesting motivation differences did not underlie performance deficits. Thus, any difference in performance across 
groups should be due to the only factor that varied consistently across groups, that is, REM restriction.

\section{Possible influences of REM restriction stress}

Stress is a confounding factor with any sleep restriction procedure. The most prevalent argument against the hypothesis that REM facilitates learning is that the stress of the REM restriction procedure results in a general cognitive impairment irrespective of learning and is unrelated to any memory processing during sleep (for review, see Horne and McGrath 1984; Vertes and Eastman 2000). Stress, or a rise in stress-related hormones such as corticosterone, can modify learning positively or negatively (for review, see Luine 1994; Sapolsky 2003; Wolf 2003), depending on the type of stressor and level of hormone rise. Several procedures were used to reduce stress in this experiment. First, multiple platforms were used to reduce immobility stress (Coenen and VanLuijtelaar 1985). This multiple platform REM restriction technique has been shown to be less stressful than the single platform technique (Coenen and Van Luijtelaar 1985). Second, the water level was well below the platform so animals' tails did not touch the water, unlike most other REM restriction studies. When animals fell or jumped into the shallow water, they were able to climb back atop the platforms without delay and were toweled dry to eliminate a thermal stress load. Third, rats had access to fresh, drinkable water at all times. Fourth, the restriction period lasted only $4 \mathrm{~h}$, which is much shorter than other studies of stress caused by REM deprivation (usually $\geq 24 \mathrm{~h}$ ) (Mendelson et al. 1974; Coenen and VanLuijtelaar 1985). Finally, the time exposed to the pedestals and the putative sleep restriction stressor were equal between the two REM-restricted groups, yet performance differed according to the timing of REM restriction. Restriction in different REM windows differentially affected spatial learning both in the present study and in that conducted by Smith and Butler (1982), although REM restriction in either window would presumably cause similar stress levels.

\section{Conclusions}

This experiment supports the idea that REM sleep is important for complex, associative learning such as tested by the eight-box spatial task. These data also support the hypothesis that the timing of REM sleep relative to training is important for some types of learning. This experiment adds to the literature by restricting REM in two windows and doing so on multiple days to determine which learning deficits caused by REM restriction are long lasting and whether the effects can ever be overcome by enough practice and/or use of alternative strategies. Significant deficits in complex associative learning continued throughout the course of chronic REM restriction. This study highlights the fact that learning strategy changes can be difficult to discern in overall performance, and careful dissection of performance after challenges such as REM restriction can make an otherwise seemingly small or temporary deficit stand out as a continuous obstruction of complex associative learning.

\section{Materials and Methods}

\section{Experimental design}

\section{Subjects and housing}

Twenty-five Fisher 344 rats aged 5 mo and weighing 250-350 g, were obtained from Harlan (Indianapolis, IN). Following acclimatization, visual acuity was tested by using the visual platform version of the Morris water maze (Morris 1984). The visual version of the Morris water maze requires animals to swim to a visible platform $2 \mathrm{~cm}$ above the water's surface as the only means of escape from a standard circular water tank. Animals performed five trials per day for two consecutive days. Only rats with performance $<2 \mathrm{SD}$ above the $2 \mathrm{~d}$ average latency to platform were deemed visually and physically fit and selected to continue the experiment. Rats were rank-ordered by average latency to platform across the $2 \mathrm{~d}$ and were divided to ensure that each group had equivalent numbers of relatively fast, moderate, and slow performers. Following completion of the Morris water maze task, rats were moved to individual standard Plexiglas cages $(45.7 \times 24.1 \times 20.3 \mathrm{~cm})$ placed inside one of three environmentally (light, sound, and temperature) controlled chambers. The housing temperature was set at $22.5 \pm 0.5^{\circ} \mathrm{C}$, with a $12: 12 \mathrm{~h}$ light/dark cycle. Testing for all animals occurred $\sim 30 \mathrm{~min}$ after light onset each day. Fresh water was available ad libitum at all times, except during performance of the behavioral task. Initially, animals were housed with corncob bedding and were able to find edible corn pieces within this bedding, so their weight was not declining and their motivation for running multiple laps each day was low: The average number of laps per day was only five for the first $5 \mathrm{~d}$. Bedding type was switched to (inedible) pine shavings from day 4 onward, and thereafter, the number of laps completed per day increased steadily.

\section{Motivation}

Ad libitum feeding was stopped prior to the initiation of training by removing food pellets. Rats only received food in the form of mash (pellets dissolved in water) during the daily 30-min training session to motivate them to run as many laps as possible in the session. However, if their weight approached the $80 \%$ free feeding minimum, they were supplemented with food mash in their home cage. By the end of the study, most rats were supplemented daily, as they were generally unable to maintain weight on the $\sim 25$ laps/d they were completing.

\section{Task description}

\section{Eight-box spatial task}

The eight-box task (Poe et al. 2002) is a rectangular raised track outfitted with eight symmetrically positioned boxes (Fig. 6).

Three of the eight-box positions were selected as goals where $\sim 0.2$ cc of food was available in a shallow food cup behind a hinged door. To encourage forward motion for accurate assessment of errors per lap, the rats were required to complete a full lap before the three boxes were rebaited. Errors were scored visually by an experimenter during the session when a rat visited a box position that never contained food (commission), slowed or stopped in front of a nonbaited box (hesitation), or ignored a baited box (omission). Since each lap was considered a new trial,

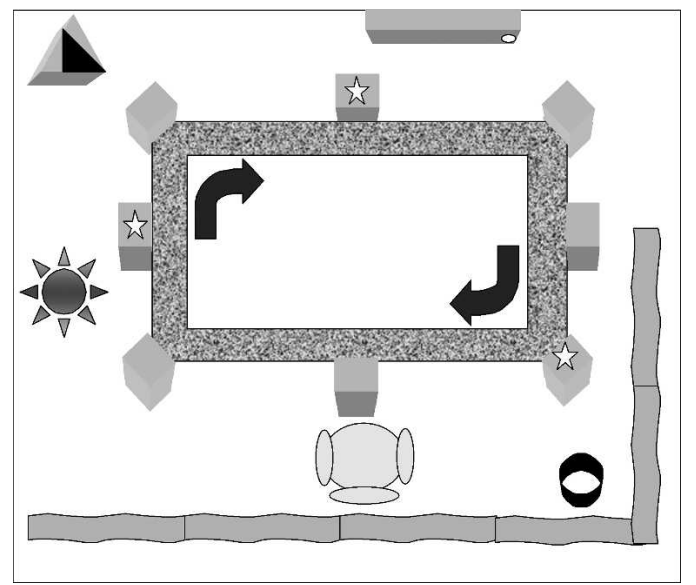

Figure 6. Overhead schematic of the eight-box task. The spatial task required rats to learn locations of food placement from three of eight available box choices on a raised rectangular track. The food-baited boxes are depicted with a star. The same three box positions were baited every lap across the $15 \mathrm{~d}$. Posters, curtains, and colored objects of different shapes (depicted by the gray symbols) surrounded the maze. All environmental cues remained constant throughout the study. 
many trials could be completed in a short period of time, allowing for the type of intensive training sessions that make the REM sleep immediately following training most germane to learning (Smith and Rose 1997). All rats were naive to the maze task prior to the first day of the experiment, so they had to learn the procedures of running for food reward as well as the spatial location of the three boxes always baited with food.

\section{Making procedural strategies less effective}

In order to increase the relative accuracy of allocentric, i.e., global referenced, spatial cues over simple intramaze cues to indicate food placement sites, the following measures were employed: (1) all boxes contained inaccessible food to equalize odor cues; (2) rats were removed from the track after every fifth lap, placed in a towel-lined shallow clay pot to rest for $2 \mathrm{~min}$, and then replaced on the track at a new start location to render inaccurate an egocentric, self-motion-related strategy such as visiting the first, third, and seventh boxes from the starting position; and (3) after every 10 laps completed (lap 10, 20, etc.) the track was rotated $180^{\circ}$ and the boxes cleaned and rebaited such that the boxes at the same room positions (not the same physical boxes) were baited for the next 10 laps. The maze rotation procedure made the positions of scent or visible cues that were left on the track unreliable relative to the places of food rewards and provided an indication of strategy used. Since the maze rotation alters any intramaze cue-to-baited box relations, but leaves all extramaze (global, spatial) cue-to-baited box relations intact, a rat relying on the simple, intramaze cues to find food rewards would show increased errors following maze rotation, while a rat relying on the spatial, extramaze cues would not be affected by rotation.

\section{Groups}

To test the contribution of REM sleep to learning on this complex associative task, we restricted six rats from entering REM sleep in the 4 -h period immediately following training (0-4 REMr). Another eight rats were returned to their home cage immediately after training to serve as normally sleeping controls. To test the specificity of the first 4-h REM window, another group of six rats was REM sleep restricted in the second 4-h period after training (4-8 REMr) and compared with the first REM-restricted group for performance.

\section{REM sleep restriction method}

The multiple platforms-over-water method (Coenen and van Luijtelaar 1985) was used to restrict REM sleep for $4 \mathrm{~h}$ each day. Three platforms of equal dimension $(6.35-\mathrm{cm}$ diameter, $24 \mathrm{~cm}$ tall) were placed next to each other in a bin (39 $\mathrm{cm}$ deep). The platforms stood above $2 \mathrm{~cm}$ of water. This water level is much lower than the standard level (level usually just below top of platforms) and prevents animals' tails from hanging in the water, reducing cold stress. The edges of the platforms were $9 \mathrm{~cm}$ apart. The rat was placed atop one of the platforms and was able to move between them, sit with its feet beneath it, and to sleep as long as it maintained enough muscle tone to hold their position. During the transition to REM sleep, antigravity muscles are inhibited (Chase and Morales 1990), causing muscle atonia (Jouvet et al. 1959), and rats begin to fall. Such strong vestibular stimulation usually awakens the animals before they actually fall off the pedestal, but if they do, the platform is low enough that they can climb back atop the pedestal themselves. These rats were not instrumented for assessing sleep/waking state. However, the multiple platform method of deprivation is relatively selective for REM sleep disruption as non-REM sleep is usually only moderately reduced $(-70 \%-85 \%$ of baseline) compared with the near total elimination of REM sleep (van Luijtelaar and Coenen 1986; Maloney et al. 1999). Behavioral observations confirm that during REM restriction, rats were generally quiescent during the 4-h platform session.

Our study had a comparable rat weight/platform diameter ratio as other studies, so we expect the same degree of REM restriction was achieved, although we cannot be sure that home cage controls did not also have reduced REM sleep compared with baseline after such an intensive learning session. Spontane- ous increases in REM sleep after spatial learning on this task (data not shown) and shown in other studies (Lucero 1970; Fishbein et al. 1974; Smith and Rose 1997) make REM reductions in controls unlikely here.

\section{Statistical analysis}

Analysis of the type and timing of errors within a training session and across days was performed to reveal the relative use of nonspatial adaptive strategies. SPSS (SPSS Inc.) statistical software package was used for all parametric analyses. Repeated-measures ANOVA was used to analyze the changes in errors per lap, performance improvement, and number of laps completed per training session between groups and days of testing. A post hoc analysis of each of three segments of the experiment was performed on the same comparisons. Segment 1 represented the first $5 \mathrm{~d}$. The second segment was the next $5 \mathrm{~d}$. And the third segment was the final $5 \mathrm{~d}$. Repeated-measures ANOVAs were performed, correcting for violation of sphericity by using the Hung-Feldt adjustment when necessary to adjust nonuniform variance across days or groups. Performance improvement was calculated by subtracting each day's mean number of errors per lap for each rat from the group average number of errors per lap on day 1 . Regression analyses were performed on the 15-d performance improvement curve, and ANCOVA analysis was used to compare improvement slope across groups. The regression and ANCOVA analyses were repeated for each 5-d segment. Regression analyses were also performed for the improvement within a day curve. The improvement within a day curve was made by averaging errors for each lap (1-30) across animals and days. The cutoff was set at lap 30 because this was the last lap which multiple animals per group completed.

To determine the difference between groups on the first lap each day, a repeated-measures ANOVA was performed. A repeated-measures ANOVA was also used to compare the average number of each type of error (commission, hesitation, omission) within groups and to test for weight differences between groups.

We measured the effect of the maze rotation (comparing errors on lap 11, after rotation, to lap 10, before rotation) on performance. The last $6 \mathrm{~d}$ of the experiment were used to compare lap 10 to lap 11 since, by then, a majority of animals were completing >11 laps consistently. A nonparametric Sign test (Freund 1999) was used since different numbers of animals contributed to the analysis each day. A Sign test was also used to compare errors made on the first lap each day between groups. Total errors on laps 1-5, 6-10, and 11-15 were binned for each rat on each day and defined as lap sets.

\section{Acknowledgments}

We would like to thank Carena Thompson, Brianna Steinhoff, and Elizabeth Ferluga for their help with the preliminary study. We thank Gilbert Jyoung and Toya Roberson for their assistance gathering behavioral data. We thank Victoria Booth for her assistance in the preparation of this manuscript. This work was supported by National Institutes of Health MH 60670 and the Department of Anesthesiology at the University of Michigan.

\section{References}

Barnes, C.A., Nadel, L., and Honig, W.K. 1980. Spatial memory deficit in senescent rats. Can. J. Psychol. 34: 29-39.

Barnes, C.A., Suster, M.S., Shen, J., and McNaughton, B.L. 1997. Multistability of cognitive maps in the hippocampus of old rats. Nature 388: 272-275.

Bonnet, M.H. and Arand, D.L. 1994. The use of prophylactic naps and caffeine to maintain performance during a continuous operation. Ergonomics 37: 1009-1020.

Borbely, A.A. 1980. Effects of light and circadian rhythm on the occurrence of REM sleep in the rat. Sleep 2: 289-298.

Borbely, A.A. and Neuhaus, H.U. 1979. Sleep-deprivation: Effects on sleep and EEG in the rat. J. Comp. Physiol. 133: 71-87.

Chase, M.H. and Morales, F.R. 1990. The atonia and myoclonia of active (REM) sleep. Annu. Rev. Psychol. 41: 557-584.

Coenen, A.M. and van Luijtelaar, E.L. 1985. Stress induced by three procedures of deprivation of paradoxical sleep. Physiol. Behav. 35: 501-504. 
De Koninck, J., Lorrain, D., Christ, G., Proulx, G., and Coulombe, D. 1989. Intensive language learning and increases in rapid eye movement sleep: Evidence of a performance factor. Int. J. Psychophysiol. 8: 43-47.

DiMattia, B.D. and Kesner, R.P. 1988. Spatial cognitive maps: Differential role of parietal cortex and hippocampal formation. Behav. Neurosci. 102: 417-480.

Dinges, D.F., Pack, F., Williams, K., Gillen, K.A., Powell, J.W., Ott, G.E., Aptowicz, C., and Pack, A.I. 1997. Cumulative sleepiness, mood disturbance, and psychomotor vigilance performance decrements during a week of sleep restricted to $4-5$ hours per night. Sleep 20: $267-277$

Drummond, S.P., Brown, G.G., Gillin, J.C., Stricker, J.L., Wong, E.C., and Buxton, R.B. 2000. Altered brain response to verbal learning following sleep deprivation. Nature 403: 655-657.

Drummond, S.P., Gillin, J.C., and Brown, G.G. 2001. Increased cerebral response during a divided attention task following sleep deprivation. J. Sleep Res. 10: 85-92.

Eckerman, D.A., Gordon, W.A., Edwards, J.D., MacPhail, R.C., and Gage, M.I. 1980. Effects of scopolamine, pentobarbital, and amphetamine on radial arm maze performance in the rat. Pharmacol. Biochem. Behav. 12: 595-602.

Eichenbaum, H. 2000. A cortical-hippocampal system for declarative memory. Nat. Rev. Neurosci. 1: 41-50.

Feinberg, I. and Floyd, T.C. 1979. Systematic trends across the night in human sleep cycles. Psychophysiology 16: 283-291.

Fishbein, W., Kastaniotis, C., and Chattman, D. 1974. Paradoxical sleep: Prolonged augmentation following learning. Brain Res. 79: 61-75.

Freund, J.E. 1999. Mathematical Statistics (eds. I. Miller and M. Miller), pp. 529-531. Simon \& Schuster, Upper Saddle River, NJ.

Greenberg, R. and Pearlman, C. 1974. Cutting the REM nerve: An approach to the adaptive role of REM sleep. Perspect. Biol. Med. 17: $513-521$.

Guan, Z., Peng, X., and Fang, J. 2004. Sleep deprivation impairs spatial memory and decreases extracellular signal-regulated kinase phosphorylation in the hippocampus. Brain Res. 1018: 38-47.

Horne, J.A. and McGrath, M.J. 1984. The consolidation hypothesis for REM sleep function: Stress and other confounding factors: A review. Biol. Psychol. 18: 164-184.

Jouvet, M., Michel, F., and Courjon, J. 1959. Sur en stade d'activite electric cerébrale rapid ou cours du sommeil physiologique. C. $R$. Seances Soc. Biol. Fil. 153: 1024-1028.

Karni, A., Tanne, D., Rubenstein, B.S., Askenasy, J.J.M., and Sagi, D. 1994. Dependence on REM sleep of overnight improvement of a perceptual task. Science 265: 679-682.

Lucero, M. 1970. Lengthening of REM sleep duration consecutive to learning in the rat. Brain Res. 20: 319-322.

Luine, V.N. 1994. Steroid hormone influences on spatial learning. Ann. N.Y. Acad. Sci. 743: 201-211.

Maloney, K.J., Mainville, L., and Jones, B.E. 1999. Differential c-Fos expression in cholingergic, monoaminergic, and GABAergic cell groups of the pontomesencephalic tegmentum after paradoxical sleep deprivation and recovery. J. Neurosci. 19: 3057-3072.

McGrath, M.J. and Cohen, D.B. 1978. REM sleep facilitation of adaptive waking behavior: A review of the literature. Psychol. Bull. 85: 24-57.

Meiri, N. and Rosenblum, K. 1998. Lateral ventricle injection of the protein synthesis inhibitor anisomycin impairs long-term memory in a spatial memory task. Brain Res. 789: 48-55.

Mendelson, W.B., Guthrie, R.D., Frederick, G., and Wyatt, R.J. 1974. The flower pot technique of rapid eye movement (REM) sleep deprivation. Pharmacol. Biochem. Behav. 2: 553-556.

Mitler, M.M., Carskadon, M.A., Czeisler, C.A., Dement, W.C., Dinges, D.F., and Graeber, R.C. 1988. Catastrophes, sleep, and public policy: Consensus report. Sleep 11: 100-109.

Morris, R. 1984. Developments of a water-maze procedure for studying spatial learning in the rat. J. Neurosci. Methods 11: 47-60.

Morris, R.G., Garrud, P., Rawlins, J.N., and O'Keefe, J. 1982. Place navigation impaired in rats with hippocampal lesions. Nature 297: 681-683.

Naghdi, N., Majlessi, N., and Bozorgmehr, T. 2003. The effects of anisomycin (a protein synthesis inhibitor) on spatial learning and memory in CA1 region of rats hippocampus. Behav. Brain Res. 139: 69-73.

Okaichi, H., Oshima, Y., and Jarrard, L.E. 1989. Scopolamine impairs both working and reference memory in rats: A replication and extension. Pharmacol. Biochem. Behav. 34: 599-602.

O'Keefe, J. and Nadel, L. 1978. The hippocampus as a cognitive map. Clarendon, Oxford, UK.

Pearlman, C.A. 1979. REM sleep and information processing: Evidence from animal studies. Neurosci. Biobehav. Rev. 3: 57-68.

Poe, G.R., Teed, R.G., Insel, N., White, R., McNaughton, B.L., and Barnes, C.A. 2000a. Partial hippocampal inactivation: Effects on spatial memory performance in aged and young rats. Behav. Neurosci. 114: 940-949.

Poe, G.R., Thompson, C.M., Ahlgren, A., and Cook, B. 2000b. REM sleep deprivation delays spatial memory task acquisition in F344 rats. Sleep 23: A17.

Poe, G.R., Thompson, C.M., Riley, B.T., Tysor, M.K., Bjorness, T.E., Steinhoff, B.P., and Ferluga, E.D. 2002. A spatial memory task appropriate for electrophysiological recordings. J. Neurosci. Methods 121: $65-74$.

Rapp, P.R., Rosenberg, R.A., and Gallagher, M. 1987. An evaluation of spatial information processing in aged rats. Behav. Neurosci. 101: $3-12$.

Ruskin, D.N., Liu, C., Dunn, K.E., Bazan, N.G., and LaHoste, G.J. 2004 Sleep deprivation impairs hippocampus-mediated contextual learning but not amygdala-mediated cued learning in rats. Eur. J. Neurosci. 19: 3121-3124.

Sapolsky, R.M. 2003. Stress and plasticity in the limbic system. Neurochem. Res. 28: 1735-1742.

Seligman, M.E. 1970. On the generality of the laws of learning. Psychol. Rev. 77: 406-418.

Shapiro, M.L., Tanila, H., and Eichenbaum, H. 1997. Cues that hippocampal place cells encode: Dynamic and hierarchical representation of local and distal stimuli. Hippocampus 7: 624-642.

Siegel, J.M. 2001. The REM sleep-memory consolidation hypothesis. Science 294: 1058-1063.

Smith, C. 1985. Sleep states and learning: A review of the animal literature. Neurosci. Biobehav. Rev. 9: 157-168.

Smith, C. and Butler, S. 1982. Paradoxical sleep at selective times following training is necessary for learning. Physiol. Behav. 29: 469-473.

Smith, C. and Rose, G.M. 1996. Evidence for a paradoxical sleep window for place learning in the Morris water maze. Physiol. Behav. 59: 93-97.

. 1997. Posttraining paradoxical sleep in rats is increased after spatial learning in the Morris water maze. Behav. Neurosci. 111: $1197-1204$.

Smith, C., Tenn, C., and Annett, R. 1991. Some biochemical and behavioral aspects of the paradoxical sleep window. Can. J. Psychol. 45: $115-124$.

Smith, C.T., Conway, J.M., and Rose, G.M. 1998. Brief paradoxical sleep deprivation impairs reference, but not working, memory in the radial arm maze task. Neurobiol. Learn. Mem. 69: 211-217.

Stickgold, R., Hobson, J.A., Fosse, R., and Fosse, M. 2001. Sleep, learning, and dreams: Off-line memory reprocessing. Science 294: 1052-1057.

Van Dongen, H.P.A., Maislin, G., Mullington, J.M., and Dinges, D.F. 2003. The cumulative cost of additional wakefulness: Dose response effects on neurobehavioral functions and sleep physiology from chronic sleep restriction and total sleep deprivation. Sleep 26: $117-126$.

van Luijtelaar, E.L. and Coenen, A.M. 1986. Electrophysiological evaluation of three paradoxical sleep deprivation techniques in rats. Physiol. Behav. 36: 603-609.

Vargha-Khadem, F., Gadian, D.G., Watkins, K.E., Connelly, A., Van Paesschen, W., and Mishkin, M. 1997. Differential effects of early hippocampal pathology on episodic and semantic memory. Science 277: $376-380$.

Vertes, R.P. and Eastman, K.M. 2000. The case against memory consolidation in REM sleep. Behav. Brain Sci. 23: 867-876.

Wesensten, N.J., Belenky, G., Kautz, M.A., Thorne, D.R., Reichardt, R.M. and Balkin, T.J. 2002. Maintaining alertness and performance during sleep deprivation: modafinil vs. caffeine. Psychopharmacology 159: 238-247.

White, N.M. and McDonald, R.J. 2002. Multiple parallel memory systems in the brain of the rat. Neurobio. Learn. Mem. 77: 125-184.

Wimmer, F., Hoffman, R.F., Bonato, R.A., and Moffitt, A.R. 1992. The effects of sleep deprivation on divergent thinking and attention processes. J. Sleep Res. 1: 223-230.

Winson, J. 1985. Brain and psyche. Anchor Press/Doubleday, New York.

Wolf, O.T. 2003. HPA axis and stress. Best Pract. Res. Clin. Endocrinol. Metab. 17: 287-299.

Wurts, S.W. and Edgar, D.M. 2000. Circadian and homeostatic control of rapid eye movement (REM) sleep: Promotion of REM tendency by the suprachiasmatic nucleus. J. Neurosci. 20: 4300-4310.

Wyatt, J.K., Cajochen, C., Ritz-De Cecco, A., Czeisler, C.A., and Dijk, D.J. 2004. Low-dose repeated caffeine administration for circadian-phase-dependent performance degradation during extended wakefulness. Sleep 27: 374-381.

Youngblood, B.D., Zhow, J., Smagin, G.N., Ryan, D.H., and Harris, R.B.S. 1997. Sleep deprivation by the "flower pot" technique and spatial reference memory. Physiol. Behav. 61: 249-256.

Received July 23, 2004; accepted in revised form February 22, 2005. 


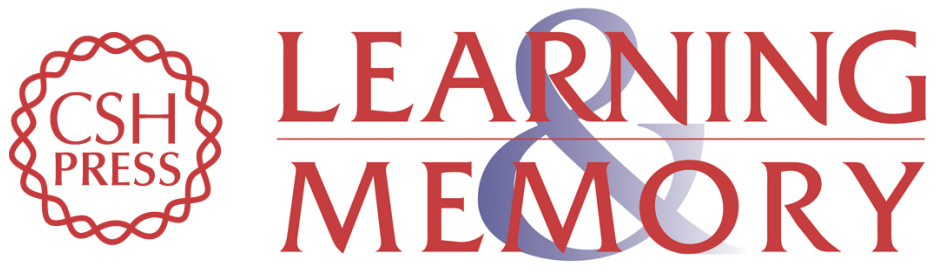

\section{REM restriction persistently alters strategy used to solve a spatial task}

Theresa E. Bjorness, Brett T. Riley, Michael K. Tysor, et al.

Learn. Mem. 2005, 12:

Access the most recent version at doi:10.1101/lm.84805

References This article cites 59 articles, 6 of which can be accessed free at:

http://learnmem.cshlp.org/content/12/3/352.full.html\#ref-list-1

License

Email Alerting Receive free email alerts when new articles cite this article - sign up in the box at the Service top right corner of the article or click here. 